\title{
On Coupled Systems of Lidstone-Type Boundary Value Problems
}

\author{
Robert de Sousa ${ }^{a, c}$, Feliz Minhós ${ }^{b, c}$ and João Fialho ${ }^{c, d}$ \\ ${ }^{a}$ Faculdade de Ciências e Tecnologia, Núcleo de Matemática e Aplicações \\ (NUMAT), Centro de Investigação em Ciências Exatas (CiCE), Universidade \\ de Cabo Verde \\ Campus de Palmarejo, 279 Praia, Cabo Verde \\ ${ }^{b}$ Departamento de Matemática, Escola de Ciências e Tecnologia \\ ${ }^{c}$ Centro de Investigação em Matemática e Aplicações (CIMA), Instituto de \\ Investigação e Formação Avançada, Universidade de Évora \\ Rua Romão Ramalho, 59, 7000-671 Évora, Portugal \\ ${ }^{d}$ Department of Mathematics, British University of Vietnam \\ Ecopark Campus, 160000 Hung Yen, Hanoi, Vietnam \\ E-mail(corresp.): robert.sousa@docente.unicv.edu.cv \\ E-mail: fminhos@uevora.pt \\ E-mail: joao.f@buv.edu.vn
}

Received June 17, 2020; revised April 12, 2021; accepted April 13, 2021

\begin{abstract}
This research concerns the existence and location of solutions for coupled system of differential equations with Lidstone-type boundary conditions. Methodology used utilizes three fundamental aspects: upper and lower solutions method, degree theory and nonlinearities with monotone conditions. In the last section an application to a coupled system composed by two fourth order equations, which models the bending of coupled suspension bridges or simply supported coupled beams, is presented.

Keywords: coupled nonlinear systems, coupled lower and upper solutions, Lidstone-type boundary value problems, operator theory, suspension bridges.
\end{abstract}

AMS Subject Classification: 34A34; 34B10; 34B15; 47N20.

\section{Introduction}

Between 1938 and 1941, the English mathematician George James Lidstone (1870-1952) published notes on interpolation, where it was shown that poly-

Copyright (c) 2021 The Author(s). Published by Vilnius Gediminas Technical University This is an Open Access article distributed under the terms of the Creative Commons Attribution License (http://creativecommons.org/licenses/by/4.0/), which permits unrestricted use, distribution, and reproduction in any medium, provided the original author and source are credited. 
nomial interpolation is the solution of boundary value problem given by

$$
\left\{\begin{array}{c}
u^{(2 m)}(t)=0, t \in[a, b] \\
u^{(j)}(a)=A_{j}, \quad u^{(j)}(b)=B_{j}, \quad j=0,2, \ldots, 2 m-2 .
\end{array}\right.
$$

The BVP (1.1) can be generalized and coupled into the following problem

$$
\begin{aligned}
u^{(2 m)}(t) & =f\left(t, u(t), u^{\prime}(t), \ldots, u^{(2 m-1)}(t)\right) t \in[0,1], \\
u^{(j)}(0) & =A_{j}, \quad u^{(j)}(1)=B_{j}, \quad j=0,2, \ldots, 2 m-2,
\end{aligned}
$$

which appears in the literature as Lidstone boundary value problems (see [17], and the references therein). It is also common to find in the literature the so called complementary Lidstone boundary value problems (see [22]),

$$
\begin{gathered}
u^{(2 m-1)}(t)=f\left(t, u(t), u^{\prime}(t), \ldots, u^{(2 m-2)}(t)\right) t \in[a, b] \\
u(a)=A_{0}, \quad u^{(2 j-1)}(a)=A_{j}, \quad u^{(2 j-1)}(b)=B_{j}, \quad j=0,1, \ldots, m-1 .
\end{gathered}
$$

Lidstone-type boundary value problems have applications in real phenomena such as the study of bending of simply-supported beams or suspended bridges (see [13, 14]). In [8], de Sousa and Minhós used Lidstone boundary conditions in a coupled system composed by two and fourth order differential equations, to model the bending of the main beam in suspension bridges. Likewise, Li and Gao [15], discuss models of a static bending elastic beam whose two ends are simply supported, given by Lidstone-type boundary conditions.

Over the course of several years, one can find several different approaches and techniques on problems or the family of problems with boundary value problems as Lidstone. For example, in [1], Agarwal and Wong deal with the existence of a positive solution of the complementary Lidstone boundary value problem

$$
\begin{gathered}
(-1)^{m} \gamma^{(2 m+1)}(t)=\lambda F\left(t, \gamma(t), \gamma^{\prime}(t)\right) t \in(0,1), \\
y(0)=0, \gamma^{(2 \kappa-1)}(0)=\gamma^{(2 \kappa-1)}(1)=0,1 \leq \kappa \leq m,
\end{gathered}
$$

where $m \geq 1, \lambda>0$, and $F$ is continuous at least in the interior of the domain of interest; in [11] the authors apply a different methodology to a similar Lidstone problem, analyzing the existence of solution via bifurcation techniques; in [3] it is studied the existence, multiplicity and nonexistence results for nontrivial solutions to a nonlinear discrete fourth-order Lidstone boundary value problem; in [6] Cid et al. consider Lidstone boundary value problem, applying the monotone iterative technique with fixed point theorems of cone expansion or compression type; in [21] the authors deal with the existence and uniqueness of solution for a class of elliptic Lidstone boundary value problems; on $[2,20]$, the authors study, respectively, Lidstone polynomials and boundary value problems and boundary layer phenomenon,... among others.

More recently on [5], Cabada and Somoza extensively study a family of problems involving Lidstone boundary conditions type. The existence of solution is proved through the usage of lower and upper solutions. 
In addition, Lidstone boundary value problems can also be found on [18], where Minhós et al. prove an existence and location result for the fourth order fully nonlinear equation

$$
u^{(i v)}=f\left(t, u, u^{\prime}, u^{\prime \prime}, u^{\prime \prime \prime}\right), \quad 0<t<1
$$

with the Lidstone boundary conditions

$$
u(0)=u(1)=u^{\prime \prime}(0)=u^{\prime \prime}(1)=0,
$$

where $f:[0,1] \times \mathbb{R}^{4} \rightarrow \mathbb{R}$ is a continuous function satisfying a Nagumo type condition.

Consequently, Fialho and Minhós in [9], study the nonlinear fully equation

$$
u^{(i v)}(x)+f\left(x, u(x), u^{\prime}(x), u^{\prime \prime}(x), u^{\prime \prime \prime}(x)\right)=0,
$$

for $x \in[0,1]$, where $f:[0,1] \times \mathbb{R}^{4} \rightarrow \mathbb{R}$ is a continuous functions, coupled with the Lidstone boundary conditions,

$$
u(0)=u(1)=u^{\prime \prime}(0)=u^{\prime \prime}(1)=0 .
$$

The authors ellaborate on how different definitions of lower and upper solutions can generalize existence and location results for boundary value problems with Lidstone boundary data.

An approach with Lidstone boundary conditions using system of differential equations can be found in [23]. The authors considered the existence of positive solutions for fourth-order nonlinear singular semipositone system

$$
\begin{aligned}
u^{(4)}(t) & =f\left(t, u(t), v(t), u^{\prime \prime}(t), v^{\prime \prime}(t)\right), \quad t \in(0,1), \\
v^{(4)}(t) & =g\left(t, u(t), v(t), u^{\prime \prime}(t), v^{\prime \prime}(t)\right), \quad t \in(0,1), \\
u(0) & =u(1)=u^{\prime \prime}(0)=u^{\prime \prime}(1)=0, \\
v(0) & =v(1)=v^{\prime \prime}(0)=v^{\prime \prime}(1)=0,
\end{aligned}
$$

with $f, g \in C((0,1) \times[0,+\infty) \times[0,+\infty) \times(-\infty, 0] \times(-\infty, 0], \mathbb{R})$. The existence results were obtained by approximating the fourth-order system to a secondorder singular one and using a fixed point index theorem on cones.

Motivated by the works mentioned above, this paper concerns the study of the fourth order coupled system

$$
\begin{cases}u^{(4)}(t)=f\left(t, u(t), v(t), u^{\prime}(t), v^{\prime}(t), u^{\prime \prime}(t), v^{\prime \prime}(t)\right), & t \in[0,1], \\ v^{(4)}(t)=h\left(t, u(t), v(t), u^{\prime}(t), v^{\prime}(t), u^{\prime \prime}(t), v^{\prime \prime}(t)\right), & t \in[0,1],\end{cases}
$$

with Lidstone boundary conditions

$$
\left\{\begin{array}{l}
u(0)=u(1)=A_{1}, \quad u^{\prime \prime}(0)=A_{2}, \quad u^{\prime \prime}(1)=A_{3}, \\
v(0)=v(1)=B_{1}, v^{\prime \prime}(0)=B_{2}, v^{\prime \prime}(1)=B_{3},
\end{array}\right.
$$

where $f, h:[0,1] \times \mathbb{R}^{6} \rightarrow \mathbb{R}$ are continuous functions and $A_{i}, B_{i} \in \mathbb{R}$, for $i=1,2,3$. 
The method applies lower and upper solutions and degree theory. The autors would like to point out, that to the best of their knowledge, it is the first time where fourth order coupled systems of differential equations with dependence of the first and second derivatives is considered subject to Lidstone boundary conditions (1.3).

This paper is outlined as follows. In Section 2, the key definitions and considerations are stated. The main theorem is presented in Section 3. Finally, in Section 4, an application to a system of coupled suspension bridges is proposed, as an example of (1.2)-(1.3), based on [16].

\section{Preliminaries}

For system (1.2)-(1.3) we consider coupled lower and upper solutions given by next definition:

Definition 1. A pair of functions $\left(\alpha_{1}, \alpha_{2}\right) \in\left(C^{4}([0,1])\right)^{2}$ is a coupled lower solution of problem (1.2)-(1.3) if

(i)

$$
\begin{aligned}
& \alpha_{1}^{(4)}(t) \leq f\left(t, \alpha_{1}(t), \alpha_{2}(t), \alpha_{1}^{\prime}(t), \alpha_{2}^{\prime}(t), \alpha_{1}^{\prime \prime}(t), \alpha_{2}^{\prime \prime}(t)\right) \\
& \alpha_{2}^{(4)}(t) \leq h\left(t, \alpha_{1}(t), \alpha_{2}(t), \alpha_{1}^{\prime}(t), \alpha_{2}^{\prime}(t), \alpha_{1}^{\prime \prime}(t), \alpha_{2}^{\prime \prime}(t)\right)
\end{aligned}
$$

(ii)

$$
\begin{aligned}
& \alpha_{1}(0) \leq A_{1}, \quad \alpha_{1}(1) \leq A_{1}, \quad \alpha_{2}(0) \leq B_{1}, \quad \alpha_{2}(1) \leq B_{1} \\
& \alpha_{1}^{\prime \prime}(0)<A_{2}, \quad \alpha_{1}^{\prime \prime}(1) \leq A_{3}, \quad \alpha_{2}^{\prime \prime}(0)<B_{2}, \quad \alpha_{2}^{\prime \prime}(1) \leq B_{3}
\end{aligned}
$$

(iii)

$$
\begin{aligned}
& \alpha_{1}^{\prime}(0)-\beta_{1}^{\prime}(0) \leq \min \left\{\beta_{1}(0)-\beta_{1}(1), \alpha_{1}(1)-\alpha_{1}(0), 0\right\} \\
& \alpha_{2}^{\prime}(0)-\beta_{2}^{\prime}(0) \leq \min \left\{\beta_{2}(0)-\beta_{2}(1), \alpha_{2}(1)-\alpha_{2}(0), 0\right\}
\end{aligned}
$$

A pair of functions $\left(\beta_{1}, \beta_{2}\right) \in\left(C^{4}[0,1]\right)^{2}$ is a coupled upper solution of problem $(1.2)-(1.3)$ if it verifies the reverse inequalities.

Remark 1. Forward, we denote $(a, b) \leq(c, d)$ meaning that $a \leq c$ and $b \leq d$, for $a, b, c, d \in \mathbb{R}$.

Remark 2. Note that if

$$
\left(\alpha_{1}^{\prime \prime}(t), \alpha_{2}^{\prime \prime}(t)\right) \leq\left(\beta_{1}^{\prime \prime}(t), \beta_{2}^{\prime \prime}(t)\right), \quad \forall t \in[0,1],
$$

then from Definition 1, (iii),

$$
\left(\alpha_{1}^{\prime}(t), \alpha_{2}^{\prime}(t)\right) \leq\left(\beta_{1}^{\prime}(t), \beta_{2}^{\prime}(t)\right), \quad \forall t \in[0,1]
$$

and from Definition 1, (ii),

$$
\left(\alpha_{1}(t), \alpha_{2}(t)\right) \leq\left(\beta_{1}(t), \beta_{2}(t)\right), \quad \forall t \in[0,1] .
$$


Below, a proposition of the degree theory that will be used to guarantee the existence of a solution for problem (1.2)-(1.3):

Proposition 1. ( $[7,10])$ Let $X$ be a Banach space, $\Omega \subset X$ be a bounded open set such that $0 \in \Omega$ and $T: \bar{\Omega} \rightarrow X$ be completely continuous linear operator such that $I-T$ is an homeomorphism. Then

$$
|d(I-T, \Omega)|=1
$$

\section{Main result}

This section is dedicated to the main theorem which is an existence and location of solution for problem (1.2)-(1.3).

Theorem 1. Let $f, h:[0,1] \times \mathbb{R}^{6} \rightarrow \mathbb{R}$ be a continuous functions. If there are coupled lower and upper solutions of (1.2)-(1.3), $\left(\alpha_{1}, \alpha_{2}\right)$ and $\left(\beta_{1}, \beta_{2}\right)$, respectively, according Definition 1, such that

$$
\left(\alpha_{1}^{\prime \prime}(t), \alpha_{2}^{\prime \prime}(t)\right) \leq\left(\beta_{1}^{\prime \prime}(t), \beta_{2}^{\prime \prime}(t)\right)
$$

and moreover, if $f$ and $h$ satisfy

$$
\begin{gathered}
f\left(t, \alpha_{1}(t), \alpha_{2}(t), \alpha_{1}^{\prime}(t), \alpha_{2}^{\prime}(t), x_{2}, \alpha_{2}^{\prime \prime}(t)\right) \geq f\left(t, x_{0}, y_{0}, x_{1}, y_{1}, x_{2}, y_{2}\right) \\
\geq f\left(t, \beta_{1}(t), \beta_{2}(t), \beta_{1}^{\prime}(t), \beta_{2}^{\prime}(t), x_{2}, \beta_{2}^{\prime \prime}(t)\right)
\end{gathered}
$$

for $t \in[0,1], \alpha_{1}^{(i)}(t) \leq x_{i} \leq \beta_{1}^{(i)}(t), i=0,1,2, \alpha_{2}^{(j)}(t) \leq y_{j} \leq \beta_{2}^{(j)}(t), j=$ $0,1,2$, and

$$
\begin{gathered}
h\left(t, \alpha_{1}(t), \alpha_{2}(t), \alpha_{1}^{\prime}(t), \alpha_{2}^{\prime}(t), \alpha_{1}^{\prime \prime}(t), y_{2}\right) \geq h\left(t, x_{0}, y_{0}, x_{1}, y_{1}, x_{2}, y_{2}\right) \\
\geq h\left(t, \beta_{1}(t), \beta_{2}(t), \beta_{1}^{\prime}(t), \beta_{2}^{\prime}(t), \beta_{1}^{\prime \prime}(t), y_{2}\right)
\end{gathered}
$$

for $t \in[0,1], \alpha_{1}^{(i)}(t) \leq x_{i} \leq \beta_{1}^{(i)}(t), i=0,1,2, \alpha_{2}^{(j)}(t) \leq y_{j} \leq \beta_{2}^{(j)}(t), j=$ $0,1,2$. Then there exists at least one nontrivial solution of (1.2)-(1.3), a pair $(u(t), v(t)) \in\left(C^{4}[0,1], \mathbb{R}\right)^{2}$ such that,

$$
\alpha_{1}^{(i)}(t) \leq u^{(i)}(t) \leq \beta_{1}^{(i)}(t), \quad \alpha_{2}^{(i)}(t) \leq v^{(i)}(t) \leq \beta_{2}^{(i)}(t),
$$

for $t \in[0,1]$ and $i=0,1,2$.

Proof. Consider the truncated, perturbed and coupled homotopic system

$$
\left\{\begin{array}{c}
u^{(4)}(t)=\lambda f\left(\begin{array}{c}
t, \delta_{10}(t, u(t)), \delta_{20}\left(t, v(t), \delta_{11}\left(t, u^{\prime}(t)\right), \delta_{21}\left(t, v^{\prime}(t),\right.\right. \\
\delta_{12}\left(t, u^{\prime \prime}(t)\right), \delta_{22}\left(t, v^{\prime \prime}(t)\right)
\end{array}\right) \\
+u^{\prime \prime}(t)-\lambda \delta_{12}\left(t, u^{\prime \prime}(t)\right) \\
v^{(4)}(t)=\lambda h\left(\begin{array}{c}
t, \delta_{10}(t, u(t)), \delta_{20}\left(t, v(t), \delta_{11}\left(t, u^{\prime}(t)\right), \delta_{21}\left(t, v^{\prime}(t),\right.\right. \\
\delta_{12}\left(t, u^{\prime \prime}(t)\right), \delta_{22}\left(t, v^{\prime \prime}(t)\right) \\
+v^{\prime \prime}(t)-\lambda \delta_{22}\left(t, v^{\prime \prime}(t)\right)
\end{array}\right)
\end{array},\right.
$$


where, for each $i=1,2, j=0,1,2, \delta_{i j}$ are the truncature functions defined by

$$
\delta_{i j}(t, w)= \begin{cases}\beta_{i}^{(j)}(t), & w>\beta_{i}^{(j)}(t) \\ w, & \alpha_{i}^{(j)}(t) \leq w \leq \beta_{i}^{(j)}(t) \\ \alpha_{i}^{(j)}(t), & w<\alpha_{i}^{(j)}(t)\end{cases}
$$

together with the boundary conditions

$$
\left\{\begin{array}{l}
u(0)=u(1)=\lambda A_{1},(1-\lambda) u^{\prime \prime \prime}(0)=\lambda\left|u^{\prime \prime}(0)-A_{2}\right|, \\
(1-\lambda) u^{\prime \prime \prime}(1)=\lambda\left|u^{\prime \prime}(1)-A_{3}\right|, \\
v(0)=v(1)=\lambda B_{1},(1-\lambda) v^{\prime \prime \prime}(0)=\lambda\left|v^{\prime \prime}(0)-B_{2}\right|, \\
(1-\lambda) v^{\prime \prime \prime}(1)=\lambda\left|v^{\prime \prime}(1)-B_{3}\right|
\end{array}\right.
$$

for $\lambda \in[0,1]$.

Step 1: There are $r_{1}, r_{1}^{*}, r_{2}, r_{2}^{*}>0$, such that every solution $(u(t), v(t))$ of problem (3.4),(3.6) satisfies

$$
\left|u^{(i)}(t)\right|<r_{1}, \quad|u(t)|<r_{1}^{*}, \quad\left|v^{(i)}(t)\right|<r_{2}, \quad|u(t)|<r_{1}^{*}, \quad \forall t \in[0,1],
$$

for $i=1,2$, independently of $\lambda \in[0,1]$. For $j=1,2$, take $r_{j}>0$ large enough such that for every $t \in[0,1]$,

$$
\begin{aligned}
& -r_{j} \leq \alpha_{j}^{\prime \prime}(t) \leq \beta_{j}^{\prime \prime}(t)<r_{j} \\
& r_{1}>\max \left\{\left|A_{2}\right|,\left|A_{3}\right|\right\}:=A, r_{1}^{*}:=r_{1}+\left|A_{1}\right|, \\
& r_{2}>\max \left\{\left|B_{2}\right|,\left|B_{3}\right|\right\}:=B, r_{2}^{*}:=r_{2}+\left|B_{1}\right|, \\
& f\left(t, \beta_{1}(t), \beta_{2}(t), \beta_{1}^{\prime}(t), \beta_{2}^{\prime}(t), \beta_{1}^{\prime \prime}(t), \beta_{2}^{\prime \prime}(t)\right)+r_{1}-\beta_{1}^{\prime \prime}(t)>0, \\
& f\left(t, \alpha_{1}(t), \alpha_{2}(t), \alpha_{1}^{\prime}(t), \alpha_{2}^{\prime}(t), \alpha_{1}^{\prime \prime}(t), \alpha_{2}^{\prime \prime}(t)\right)-r_{1}-\alpha_{1}^{\prime \prime}(t)<0, \\
& f\left(t, \beta_{1}(t), \beta_{2}(t), \beta_{1}^{\prime}(t), \beta_{2}^{\prime}(t), \beta_{1}^{\prime \prime}(t), \beta_{2}^{\prime \prime}(t)\right)+r_{2}-\beta_{2}^{\prime \prime}(t)>0, \\
& h\left(t, \alpha_{1}(t), \alpha_{2}(t), \alpha_{1}^{\prime}(t), \alpha_{2}^{\prime}(t), \alpha_{1}^{\prime \prime}(t), \alpha_{2}^{\prime \prime}(t)\right)-r_{2}-\alpha_{2}^{\prime \prime}(t)<0 .
\end{aligned}
$$

Assume that, by contradiction, for $i=2$ there exists $t_{0} \in[0,1]$, such that

$$
\left|u^{\prime \prime}\left(t_{0}\right)\right| \geq r_{1}
$$

In the case $u^{\prime \prime}\left(t_{0}\right) \geq r_{1}$ define

$$
\max _{t \in[0,1]} u^{\prime \prime}(t):=u^{\prime \prime}\left(t_{0}\right) \geq r_{1}
$$

(I) If $t_{0} \in(0,1)$, we have $u^{\prime \prime \prime}\left(t_{0}\right)=0$ and $u^{(4)}\left(t_{0}\right) \leq 0$. Therefore, for $\lambda=0$ and by (3.4), (3.5), the following contradiction holds

$$
0 \geq u^{(4)}\left(t_{0}\right)=u^{\prime \prime}\left(t_{0}\right) \geq r_{1}>0
$$

(II) On the other hand, if $\lambda \in(0,1]$ and by $(3.1),(3.4),(3.5),(3.8)$, and (3.10), 
we have the following contradiction

$$
\begin{aligned}
& 0 \geq u^{(4)}\left(t_{0}\right)=\lambda f\left(\begin{array}{c}
t_{0}, \delta_{10}\left(t_{0}, u\left(t_{0}\right)\right), \delta_{20}\left(t_{0}, v\left(t_{0}\right)\right), \delta_{11}\left(t_{0}, u^{\prime}\left(t_{0}\right)\right), \\
\delta_{21}\left(t_{0}, v^{\prime}\left(t_{0}\right)\right), \beta_{1}^{\prime \prime}\left(t_{0}\right), \delta_{22}\left(t_{0}, v^{\prime \prime}\left(t_{0}\right)\right)
\end{array}\right) \\
& +u^{\prime \prime}\left(t_{0}\right)-\lambda \beta_{1}^{\prime \prime}\left(t_{0}\right) \\
& \geq \lambda f\left(t_{0}, \beta_{1}\left(t_{0}\right), \beta_{2}\left(t_{0}\right), \beta_{1}^{\prime}\left(t_{0}\right), \beta_{2}^{\prime}\left(t_{0}\right), \beta_{1}^{\prime \prime}\left(t_{0}\right), \beta_{2}^{\prime \prime}\left(t_{0}\right)\right)+u^{\prime \prime}\left(t_{0}\right)-\lambda \beta_{1}^{\prime \prime}\left(t_{0}\right) \\
& =\lambda\left[\begin{array}{c}
f\left(t_{0}, \beta_{1}\left(t_{0}\right), \beta_{2}\left(t_{0}\right), \beta_{1}^{\prime}\left(t_{0}\right), \beta_{2}^{\prime}\left(t_{0}\right), \beta_{1}^{\prime \prime}\left(t_{0}\right), \beta_{2}^{\prime \prime}\left(t_{0}\right)\right) \\
+r_{1}-\beta_{1}^{\prime \prime}\left(t_{0}\right)
\end{array}\right]>0 .
\end{aligned}
$$

If $t_{0}=0$, that is

$$
\max _{t \in[0,1]} u^{\prime \prime}(t):=u^{\prime \prime}(0) \geq r_{1}>A_{2}
$$

then $u^{\prime \prime \prime}(0) \leq 0$.

If $\lambda=0$, we have $u^{\prime \prime \prime}(0)=0, u^{(4)}(0) \leq 0$ and the arguments follow as in the previous case for interior points.

If $\lambda \in(0,1]$, by (3.6), (3.9) and (3.11), this contradiction holds

$$
0 \geq(1-\lambda) u^{\prime \prime \prime}(0)=\lambda\left|u^{\prime \prime}(0)-A_{2}\right| \geq \lambda\left(r_{1}-A_{2}\right)>0 .
$$

So, $\left|u^{\prime \prime}(t)\right|<r_{1}$. Applying the similar method above, it may be shown that $\left|v^{\prime \prime}(t)\right|<r_{2}$, for $r_{2}$ verifying (3.9) and (3.10).

By (1.3) and Rolle's theorem there exists $\xi \in(0,1)$, such that $u^{\prime}(\xi)=0$. Therefore,

$$
\begin{aligned}
& \left|u^{\prime}(t)\right| \leq \int_{\xi}^{t}\left|u^{\prime \prime}(s)\right| d s \leq r_{1}(t-\xi) \leq r_{1} \\
& |u(t)|=\left|u(0)+\int_{0}^{t} u^{\prime}(s) d s\right| \leq\left|A_{1}\right|+r_{1} t \leq r_{1}+\left|A_{1}\right|:=r_{1}^{*} .
\end{aligned}
$$

Similarly, it can be proved that $\left|v^{\prime}(t)\right|<r_{2}$ and $|v(t)|<r_{2}^{*}$.

Step 2: For $\lambda=1$ the system (3.4), (3.6) has a least solution $(u(t), v(t))$.

Define the operators $\mathcal{F}: C^{4}([0,1]) \rightarrow C([0,1]) \times \mathbb{R}^{4}, \mathcal{H}: C^{4}([0,1]) \rightarrow$ $C([0,1]) \times \mathbb{R}^{4}$ by

$$
\mathcal{F} u=\left(u^{(4)}, u(0), u(1), u^{\prime \prime}(0), u^{\prime \prime}(1)\right), \mathcal{H} v=\left(v^{(4)}, v(0), v(1), v^{\prime \prime}(0), v^{\prime \prime}(1)\right)
$$

and for $\lambda \in[0,1]$ operators $\mathcal{N}_{\lambda}, \mathcal{N}_{\lambda}^{*}: C^{3}([0,1]) \rightarrow C([0,1]) \times \mathbb{R}^{4}$ by

$$
\begin{aligned}
& \mathcal{N}_{\lambda} u=\left(\begin{array}{c}
\lambda f\left(\begin{array}{c}
t, \delta_{10}(t, u(t)), \delta_{20}(t, v(t)), \delta_{11}\left(t, u^{\prime}(t)\right), \\
\delta_{21}\left(t, v^{\prime}(t)\right), \delta_{12}\left(t, u^{\prime \prime}(t)\right), \delta_{22}\left(t, v^{\prime \prime}(t)\right)
\end{array}\right)+\mu_{1}(u), \\
\lambda A_{1}, \lambda A_{1}, \lambda\left(u^{\prime \prime \prime}(0)+\left|u^{\prime \prime}(0)-A_{2}\right|\right), \lambda\left(u^{\prime \prime \prime}(1)+\left|u^{\prime \prime}(1)-A_{3}\right|\right)
\end{array}\right), \\
& \mathcal{N}_{\lambda}^{*} v=\left(\begin{array}{c}
\lambda h\left(\begin{array}{c}
t, \delta_{10}(t, u(t)), \delta_{20}(t, v(t)), \delta_{11}\left(t, u^{\prime}(t)\right), \\
\delta_{21}\left(t, v^{\prime}(t)\right), \delta_{12}\left(t, u^{\prime \prime}(t)\right), \delta_{22}\left(t, v^{\prime \prime}(t)\right)
\end{array}\right)+\mu_{2}(v), \\
\lambda B_{1}, \lambda B_{1}, \lambda\left(v^{\prime \prime \prime}(0)+\left|v^{\prime \prime}(0)-B_{2}\right|\right), \lambda\left(v^{\prime \prime \prime}(1)+\left|v^{\prime \prime}(1)-B_{3}\right|\right)
\end{array}\right) .
\end{aligned}
$$

where $\mu_{1}(u)=u^{\prime \prime}(t)-\lambda \delta_{12}\left(t, u^{\prime \prime}(t)\right), \mu_{2}(v)=v^{\prime \prime}(t)-\lambda \delta_{22}\left(t, v^{\prime \prime}(t)\right)$. 
As $\mathcal{F}, \mathcal{H}$ have a compact inverses we can define the completely continuous operator

$$
\mathcal{T}_{\lambda}, \mathcal{T}_{\lambda}^{*}:\left(C^{3}([0,1]), \mathbb{R}\right) \rightarrow\left(C^{3}([0,1]), \mathbb{R}\right)
$$

given by $\mathcal{T}_{\lambda} u=\left(\mathcal{F}^{-1} \mathcal{N}_{\lambda}\right) u$ and $\mathcal{T}_{\lambda}^{*} v=\left(\mathcal{H}^{-1} \mathcal{N}_{\lambda}^{*}\right) v$.

Define the set

$$
\Omega=\left\{\begin{array}{c}
(u, v) \in C^{3}([0,1]):\left\|u^{(i)}\right\|_{\infty}<r_{1},\|u\|_{\infty}<r_{1}^{*}, \\
\left\|v^{(i)}\right\|_{\infty}<r_{2},\|v\|_{\infty}<r_{2}^{*}, i=1,2
\end{array}\right\},
$$

where $r_{i}$ and $r_{i}^{*}$ are given by Step 1 . So, $d\left(I-\mathcal{T}_{\lambda}, \Omega, 0\right)$ and $d\left(I-\mathcal{T}_{\lambda}^{*}, \Omega, 0\right)$ are well defined for every $\lambda \in[0,1]$. The arguments follow for $\mathcal{T}_{\lambda}$, as for $\mathcal{T}_{\lambda}^{*}$ they are similar.

By the invariance under homotopy,

$$
d\left(I-\mathcal{T}_{0}, \Omega, 0\right)=d\left(I-\mathcal{T}_{1}, \Omega, 0\right) .
$$

The equation $\mathcal{T}_{0}(u)=u$ is equivalent to the problem

$$
\left\{\begin{array}{l}
u^{(4)}(t)=u^{\prime \prime}(t), \\
u(0)=u(1)=u^{\prime \prime \prime}(0)=u^{\prime \prime \prime}(1)=0,
\end{array}\right.
$$

which has only the null solution. Then by Proposition 1,

$$
\left|d\left(I-\mathcal{T}_{0}, \Omega, 0\right)\right|=1,
$$

and consequently $\mathcal{T}_{1}(u)=u$ has at least one solution. So, the problem (3.4), (3.6) has at least one solution $(u(t), v(t))$ in $\Omega$, for $\lambda=1$.

Step 3: This solution $(u(t), v(t))$ is a solution of the system (1.2)-(1.3), too, if

$$
\alpha_{1}^{(i)}(t) \leq u^{(i)}(t) \leq \beta_{1}^{(i)}(t), \quad \alpha_{2}^{(i)}(t) \leq v^{(i)}(t) \leq \beta_{2}^{(i)}(t),
$$

for $t \in[0,1]$ and $i=0,1,2$.

Suppose by contradiction, that there is $t \in[0,1]$ such that $\alpha_{1}^{\prime \prime}(t)>u^{\prime \prime}(t)$ and consider

$$
\min _{t \in[0,1]}\left[u^{\prime \prime}(t)-\alpha_{1}^{\prime \prime}(t)\right]:=u^{\prime \prime}\left(t_{0}\right)-\alpha_{1}^{\prime \prime}\left(t_{0}\right)<0 .
$$

In fact $t_{0} \neq 0$, as, by (1.3) and Definition 1 (ii),

$$
u^{\prime \prime}(0)-\alpha_{1}^{\prime \prime}(0)=A_{2}-\alpha_{1}^{\prime \prime}(0)>0 .
$$

Analogously $t_{0} \neq 1$.

For $\left.t_{0} \in\right] 0,1\left[\right.$, then $u^{\prime \prime \prime}\left(t_{0}\right)-\alpha_{1}^{\prime \prime \prime}\left(t_{0}\right)=0$ and $u^{(4)}\left(t_{0}\right) \geq \alpha_{1}^{(4)}\left(t_{0}\right)$. So, by Definition 1 and (3.1), we have the following contradiction

$$
\begin{aligned}
& u^{(4)}\left(t_{0}\right)=f\left(\begin{array}{c}
t_{0}, \delta_{10}\left(t_{0}, u\left(t_{0}\right)\right), \delta_{20}\left(t_{0}, v\left(t_{0}\right)\right), \delta_{11}\left(t_{0}, u^{\prime}\left(t_{0}\right)\right), \\
\delta_{21}\left(t_{0}, v^{\prime}\left(t_{0}\right)\right), \alpha_{1}^{\prime \prime}\left(t_{0}\right), \delta_{22}\left(t_{0}, v^{\prime \prime}\left(t_{0}\right)\right)
\end{array}\right) \\
& \quad+u^{\prime \prime}\left(t_{0}\right)-\alpha_{1}^{\prime \prime}\left(t_{0}\right) \\
& \leq f\left(t_{0}, \alpha_{1}\left(t_{0}\right), \alpha_{2}\left(t_{0}\right), \alpha_{1}^{\prime}\left(t_{0}\right), \alpha_{2}^{\prime}\left(t_{0}\right), \alpha_{1}^{\prime \prime}\left(t_{0}\right), \alpha_{2}^{\prime \prime}\left(t_{0}\right)\right)+u^{\prime \prime}\left(t_{0}\right)-\alpha_{1}^{\prime \prime}\left(t_{0}\right) \\
& <f\left(t_{0}, \alpha_{1}\left(t_{0}\right), \alpha_{2}\left(t_{0}\right), \alpha_{1}^{\prime}\left(t_{0}\right), \alpha_{2}^{\prime}\left(t_{0}\right), \alpha_{1}^{\prime \prime}\left(t_{0}\right), \alpha_{2}^{\prime \prime}\left(t_{0}\right)\right) \leq \alpha_{1}^{\prime \prime}\left(t_{0}\right) .
\end{aligned}
$$


Therefore, $\alpha^{\prime \prime}(t) \leq u^{\prime \prime}(t)$ for $t \in[0,1]$, and by similar arguments it can be proved that $u^{\prime \prime}(t) \leq \beta_{1}^{\prime \prime}(t)$, for $t \in[0,1]$, and the correspondent inequalities for $v(t)$. Therefore,

$$
\alpha_{1}^{\prime \prime}(t) \leq u^{\prime \prime}(t) \leq \beta_{1}^{\prime \prime}(t), \quad \alpha_{2}^{\prime \prime}(t) \leq v^{\prime \prime}(t) \leq \beta_{2}^{\prime \prime}(t), \quad \forall t \in[0,1] .
$$

By integration of (3.12), (1.3), Definition 1 (iii), and (3.1), we have

$$
\alpha_{1}^{(i)}(t) \leq u^{(i)}(t) \leq \beta_{1}^{(i)}(t), \quad \alpha_{2}^{(i)}(t) \leq v^{(i)}(t) \leq \beta_{2}^{(i)}(t),
$$

for $t \in[0,1]$ and $i=0,1,2$.

Remark 3. In Definition 1, the strict inequalities in assumption (ii) imply that lower and upper functions can not be constants, which is crucial for Theorem 1 . This is highlighted by the following counter example:

Example 1. Consider the problem

$$
\left\{\begin{array}{c}
u^{(4)}(t)=-v(t)+\sin t, \text { for } t \in(0,1) \\
v^{(4)}(t)=-u(t)+\cos t, \text { for } t \in(0,1) \\
u(0)=u(1)=u^{\prime \prime}(0)=u^{\prime \prime}(1)=0 \\
v(0)=v(1)=v^{\prime \prime}(0)=v^{\prime \prime}(1)=0
\end{array}\right.
$$

Assume that, in Definition 1, assumption (ii) is replaced by

$$
\begin{aligned}
& \alpha_{1}(0) \leq A_{1}, \quad \alpha_{2}(0) \leq B_{1}, \quad \alpha_{1}(1) \leq A_{1}, \quad \alpha_{2}(1) \leq B_{1}, \\
& \alpha_{1}^{\prime \prime}(0) \leq A_{2}, \quad \alpha_{2}^{\prime \prime}(0) \leq B_{2}, \quad \alpha_{1}^{\prime \prime}(1) \leq A_{3}, \quad \alpha_{2}^{\prime \prime}(1) \leq B_{3} .
\end{aligned}
$$

Then, the pairs of constant functions

$$
\left(\alpha_{1}, \alpha_{2}\right)=(-1,-1), \quad\left(\beta_{1}, \beta_{2}\right)=(1,1)
$$

are coupled lower and upper solutions of problem (3.13) according Definition 1, for

$$
A_{1}=B_{1}=A_{2}=A_{3}=B_{2}=B_{3}=0 .
$$

The functions

$$
\begin{aligned}
& f\left(t, x_{0}, y_{0}, x_{1}, y_{1}, x_{2}, y_{2}\right)=-y_{0}+\sin t, \\
& h\left(t, x_{0}, y_{0}, x_{1}, y_{1}, x_{2}, y_{2}\right)=-x_{0}+\cos t
\end{aligned}
$$

satisfy the monotone assumptions (3.1) and (3.2). Then, by Theorem 1 , system (3.13) would have at least one solution $(u, v) \in\left(C^{4}([0,1])\right)^{2}$, such that,

$$
-1 \leq u(t) \leq 1, \quad-1 \leq v(t) \leq 1, \forall t \in[0,1] .
$$

However, from (3.3), this solution must be constant and, from the boundary conditions of (3.13), it would be the trivial solution $(u, v)=(0,0)$, which is not a solution of (3.13). Therefore, the strict inequalities in Definition 1, assumption (ii), can not be removed. 


\section{Bending of crossed suspension bridges}

In this section a coupled system composed by two suspension crossed bridges is considered. To comply with the conceptual meaning of the model, the authors will adopt $x$ as independent variable for this section, instead of $t$, as previously, as in these models, as $x$ represents displacement. Such suspension crossed bridges can be approached via a coupled system of two fourth order differential equations, following the same principle as the original model suggested in [12], assuming the adapted form

$$
\begin{aligned}
& E_{1} I_{1} w_{1}^{(4)}(x)+H_{1}\left(\frac{w_{1}^{\prime}(x)+w_{2}^{\prime}(x)}{1+\left(y_{1}^{\prime}(x)\right)^{2}}\right) \\
& \quad+\left(\left|w_{1}^{\prime \prime}(x)\right| w_{2}^{\prime \prime}(x)+y_{1}^{\prime}(x)\right)+\left(w_{1}(x)\right)^{3}=p_{1}(x), \text { for } x \in\left(0, L_{1}\right), \\
& E_{2} I_{2} w_{2}^{(4)}(x)+H_{2}\left(\frac{w_{1}^{\prime}(x)+w_{2}^{\prime}(x)}{1+\left(y_{2}^{\prime}(x)\right)^{2}}\right) \\
& \quad+\left(w_{1}^{\prime \prime}(x)\left|w_{2}^{\prime \prime}(x)\right|+y_{2}^{\prime}(x)\right)+\left(w_{2}(x)\right)^{3}=p_{2}(x), \text { for } x \in\left(0, L_{2}\right), \\
& w_{1}(0)=w_{1}\left(L_{1}\right)=w_{1}^{\prime \prime}(0)=w_{1}^{\prime \prime}\left(L_{1}\right)=0, \\
& w_{2}(0)=w_{2}\left(L_{2}\right)=w_{2}^{\prime \prime}(0)=w_{2}^{\prime \prime}\left(L_{2}\right)=0,
\end{aligned}
$$

where for $i=1,2, H_{i}, p_{i}$, are non negative forces, $w_{i}=w_{i}(x)$ are the vertical displacement of the beams, $y_{i}=y_{i}(x)$ are the position of the cable at rest, $E_{i}$ and $I_{i}$ are the elastic modulus of the material and the moment of inertia of the cross section respectively, combined they are the flexural rigidity related with the material used on each bridge. $H_{i}$ are the horizontal tensions in each cable, subject to some loads $p_{i}=p_{i}(x)$ and $L_{i}$ are the lengths of the respective beams.

As an example, the potential type of bridge described can be visualized in the diagram of Figure 1. This example stresses on the level of applicability of the theory developed.

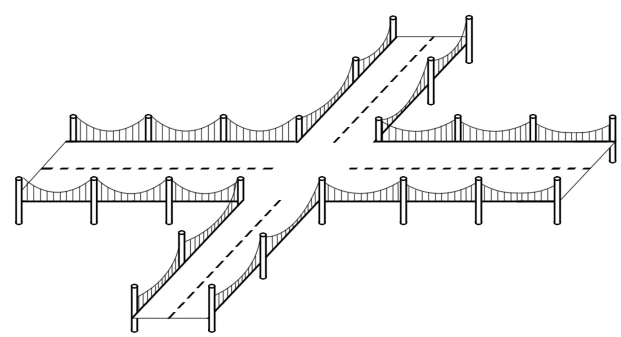

Figure 1. Diagram coupled bridges.

As a numeric example, we consider in (4.1) the values $E_{1} I_{1}=3.93 \times 10^{8} \mathrm{~N} . \mathrm{m}$, $E_{2} I_{2}=3.83 \times 10^{8}$ N.m, $H_{1}=3.4, H_{2}=2.5, p_{1}(x)=L_{1} \sin (x), p_{2}(x)=$ 
$L_{2} \sin (x), L_{1}=1, L_{2}=1$ also $y_{1}(x)=3\left(x-\frac{L_{1}}{2}\right)^{2}$ and $y_{2}(x)=4\left(x-\frac{L_{2}}{2}\right)^{2}$, that is, (4.1) becomes

$$
\begin{aligned}
& 3.93 \times 10^{8} w_{1}^{(4)}(x)+3.4\left(\frac{w_{1}^{\prime}(x)+w_{2}^{\prime}(x)}{1+(6 x-3)^{2}}\right)+\left|w_{1}^{\prime \prime}(x)\right| w_{2}^{\prime \prime}(x) \\
& -6 x+3+\left(w_{1}(x)\right)^{3}=\sin (x), \text { for } x \in(0,1), \\
& 3.83 \times 10^{8} w_{2}^{(4)}(x)+2.5\left(\frac{w_{1}^{\prime}(x)+w_{2}^{\prime}(x)}{1+(8 x-4)^{2}}\right)+w_{1}^{\prime \prime}(x)\left|w_{2}^{\prime \prime}(x)\right| \\
& \quad+8 x-4+\left(w_{2}(x)\right)^{3}=\sin (x), \text { for } x \in(0,1), \\
& w_{1}(0)=w_{1}(1)=w_{1}^{\prime \prime}(0)=w_{1}^{\prime \prime}(1)=0, \\
& w_{2}(0)=w_{2}(1)=w_{2}^{\prime \prime}(0)=w_{2}^{\prime \prime}(1)=0 .
\end{aligned}
$$

The flexural rigidity values considered were based on [4]. It is clear to see that (4.2) is a particular case of (1.2), where

$$
\begin{aligned}
& f\left(x, x_{0}, y_{0}, x_{1}, y_{1}, x_{2}, y_{2}\right)=\frac{1}{3.93 \times 10^{8}} \\
& \quad \times\left(-3.4\left(\frac{x_{1}+y_{1}}{1+(6 x-3)^{2}}\right)-\left|x_{2}\right| y_{2}-6 x+3-\left(x_{0}\right)^{3}+p_{1}(x)\right), \\
& h\left(x, x_{0}, y_{0}, x_{1}, y_{1}, x_{2}, y_{2}\right)=\frac{1}{3.83 \times 10^{8}} \\
& \quad \times\left(-2.5\left(\frac{x_{1}+y_{1}}{1+(8 x-4)^{2}}\right)-x_{2}\left|y_{2}\right|-8 x+4-\left(y_{0}\right)^{3}+p_{2}(x)\right) .
\end{aligned}
$$

For this case, all conditions in Theorem 1 are satisfied and the pairs

$$
\left(\alpha_{1}, \alpha_{2}\right)=\left(-x^{2}-x,-2 x^{2}-2 x\right), \quad\left(\beta_{1}, \beta_{2}\right)=\left(x^{2}+x, 2 x^{2}+2 x\right)
$$

are coupled lower and upper solutions of problem (4.2) according Definition 1.

In addition the nonlinearities, (4.3) satisfy all the monotone assumptions, (3.1), therefore, by Theorem 1, the system (4.2) has at least one non trivial solution $\left(w_{1}, w_{2}\right) \in\left(C^{4}([0,1])\right)^{2}$, that is,

$$
\begin{aligned}
& -x^{2}-x \leq w_{1}(x) \leq x^{2}+x, \quad \forall x \in[0,1], \\
& -2 x^{2}-2 x \leq w_{2}(x) \leq 2 x^{2}+2 x, \quad \forall x \in[0,1] .
\end{aligned}
$$

The authors would like to highlight that this solution is in fact a non-trivial solution, as the null function does not satisfy (4.2).

Moreover, we can estimate graphically the bending of both suspension bridges, as it is illustrated in the Figure 2.

\section{Conclusions}

In this paper the authors study and present an existence and location result for the problem (1.2)-(1.3) using the upper and lower solutions method. One 


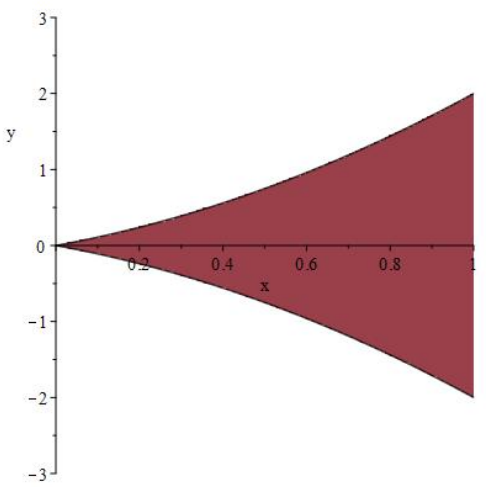

(a) Area for $w_{1}(x)$.

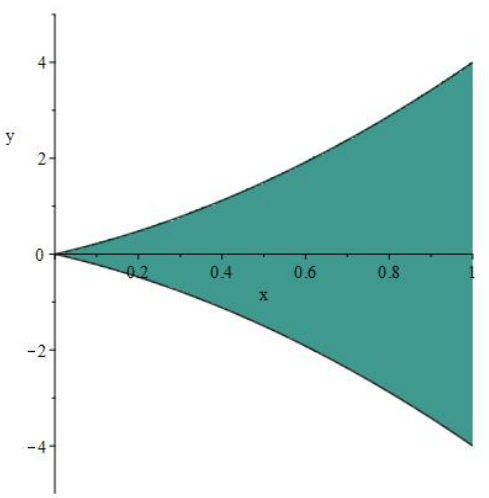

(b) Area for $w_{2}(x)$.

Figure 2. Localization of solutions $w_{1}(x)$ and $w_{2}(x)$.

of the key features of the method used lies on the extra-information that can be obtained on the solution. In fact, in addition to the existence of solution, the upper and lower solution provide details on the solution's location and also on it's derivatives. This type of information can, therefore provide some qualitative insight on the solution.

To highlight the level of applicability of the results shown, an application to crossed suspended bridges is presented, following some adjustments on the models suggested in [12].

Our method can be adapted to fourth order coupled systems with fully differential equations, applying the Nagumo condition to control the third derivatives, following the method suggested in [19].

To generalize into a fully higher order Lidstone problem it remains to find how to define lower and upper solutions to have some relation (well order or reverse order) on the odd derivatives of lower and upper solutions.

\section{References}

[1] R.P. Agarwal and P.J. Wong. Eigenvalues of complementary Lidstone boundary value problems. Boundary Value Problems, 2012(49), 2012. https://doi.org/10.1186/1687-2770-2012-49.

[2] R.P. Agarwal and P.J.Y. Wong. Lidstone polynomials and boundary value problems. Computers \& Mathematics with Applications, 17(10):1397-1421, 1989. https://doi.org/10.1016/0898-1221(89)90023-0.

[3] D.R. Anderson and F. Minhós. A discrete fourth-order Lidstone problem with parameters. Applied Mathematics and Computation, 214(2):523-533, 2009. https://doi.org/10.1016/j.amc.2009.04.034.

[4] A. Bagheri, M. Alipour, O.E. Ozbulut and D.K. Harris. Identification of flexural rigidity in bridges with limited structural information. Journal of Structural Engineering, 144(8):04018126, 2018. https://doi.org/10.1061/(ASCE)ST.1943$541 X .0002131$. 
[5] A. Cabada and L. López-Somoza. Lower and upper solutions for even order boundary value problems. Mathematics, $\mathbf{7}(10): 878,2019$. https://doi.org/10.3390/math7100878.

[6] J.A. Cid, D. Franco and F. Minhós. Positive fixed points and fourth-order equations. Bulletin of the London Mathematical Society, 41(1):72-78, 2009. https://doi.org/10.1112/blms/bdn105.

[7] C. de Coster and P. Habets. Two-Point Boundary Value Problems: Lower and Upper Solutions, volume 205. Elsevier Science \& Technology, Oxford, United Kingdom, 2006.

[8] R. de Sousa and F. Minhós. Coupled systems of Hammerstein-type integral equations with sign-changing kernels. Nonlinear Analysis: Real World Applications, 50:469-483, 2019. https://doi.org/10.1016/j.nonrwa.2019.05.011.

[9] J. Fialho and F. Minhós. The role of lower and upper solutions in the generalization of Lidstone problems. Discrete and Continuous Dynamical Systems, 2013(Special):217-226, 2013. https://doi.org/10.3934/proc.2013.2013.217.

[10] P. Fitzpatrick, M. Martelli, J. Mawhin and R. Nussbaum. Theory of Ordinary Differential Equations. Springer-Verlag Berlin Heidelberg, 1993. https://doi.org/10.1007/BFb0085073.

[11] C. Gao and J. Xu. Bifurcation techniques and positive solutions of discrete Lidstone boundary value problems. Applied Mathematics and Computation, 218(2):434-444, 2011. https://doi.org/10.1016/j.amc.2011.05.083.

[12] F. Gazzola, Y. Wang and R. Pavani. Variational formulation of the Melan equation. Mathematical Methods in the Applied Sciences, 41(3):943-951, 2016. https://doi.org/10.1002/mma.3962.

[13] C.P. Gupta. Existence and uniqueness results for the bending of an elastic beam equation at resonance. Journal of Mathematical Analysis and Applications, 135(1):208-225, 1988. https://doi.org/10.1016/0022-247X(88)90149-7.

[14] C.P. Gupta. Existence and uniqueness theorems for the bending of an elastic beam equation. Applicable Analysis: An International Journal, 26(4):289-304, 2007. https://doi.org/10.1080/00036818808839715.

[15] Y. Li and Y. Gao. Existence and uniqueness results for the bending elastic beam equations. Applied Mathematics Letters, 95:72-77, 2019. https://doi.org/10.1016/j.aml.2019.03.025.

[16] J. Melan. Theory of arches and suspension bridges. Myron Clark Pul. Comp., London, 1913.

[17] F. Minhós and H. Carrasco. Higher Order Boundary Value Problems on Unbounded Domains. World Scientific Publishing Company, Singapore, 2017. https://doi.org/10.1142/10448.

[18] F. Minhós, T. Gyulov and A.I. Santos. Existence and location result for a fourth order boundary value problem. Discrete and Continuous Dynamical Systems, 2005(0133-0189_2005_Special_662):662-671, 2005.

[19] L. Sun, M. Zhou and G. Wang. Existence and location results for fully nonlinear boundary value problem of $n$ th-order nonlinear system. Boundary Value Problems, 2009(791548):17, 2009. https://doi.org/10.1155/2009/791548.

[20] R. Vrabel. Formation of boundary layers for singularly perturbed fourthorder ordinary differential equations with the Lidstone boundary conditions. Journal of Mathematical Analysis and Applications, 440(1):65-73, 2016. https://doi.org/10.1016/j.jmaa.2016.03.017. 
[21] Y.-M. Wang. Higher-order Lidstone boundary value problems for elliptic partial differential equations. Journal of Mathematical Analysis and Applications, 308(1):314-333, 2005. https://doi.org/10.1016/j.jmaa.2005.01.019.

[22] P.J.Y. Wong. Triple solutions of complementary Lidstone boundary value problems via fixed point theorems. Boundary Value Problems, 125(2014), 2014. https://doi.org/10.1186/1687-2770-2014-125.

[23] F. Zhu, L. Liu and Y. Wu. Positive solutions for systems of a nonlinear fourth-order singular semipositone boundary value problems. Applied Mathematics and Computation, 216(2):448-457, 2010. https://doi.org/10.1016/j.amc.2010.01.038. 